\title{
ILLUSIONS OF REVERSIBLE PERSPECTIVE.
}

BY DR. ANNA VICHOLKOVSKA.

Lezeris Institute, Chicago.

This is a sketch of a work concerning the optical inversion that is observable in geometric figures and perspective designs. The special characteristic of these figures is that they call into consciousness two or more different interpretations. Thus for instance, if one fixes the point of intersection of two straight lines of a hexahedron he perceives it with one of its surfaces either inclined downward or lifted up, by a corresponding change of the position of the axis.

The illusory phenomena observed in a great many such figures have been explained by three different kinds of theories, namely by psychological, physiological, and psycho-physical theories.

I. Psychological explanations of the phenomenon are mostly generalizing conceptions, according to which inversion is a product of will, imagination, false judgment, or attention. Weadstone, ${ }^{1}$ for instance, in his critique of the physiological theory of Necker, maintains that inversion is an effect of intuition. Hering, ${ }^{2}$ in his 'Physiological Optics' says that the question of what kind of inversion will appear is largely a matter of chance or volition. Helmholtz asserts the same thing. He says that the perception of perspective figures has its cause in imagination and especially in the images of memory. ${ }^{3}$ If I imagine vividly one or the other form of the figure its apparition is imminent. Or "we can also produce intentionally a change of inversion, if we recall vividly the image of its contrary form. When its resemblance to that of the figure looked at is established, then the perspective remains stationary without effort." Helmholtz

1 Poggendorfs Annalen, I., 5 I.

${ }^{2}$ Hermann, Handbuch der Physiologie, 580.

'Physiologische Optik, 771-777.

276 
insists that: "Glancing at a figure we observe spontaneously one or the other form of perspective and usually the one that is associated in our memory with the greatest number of images." But Helmholtz and Hering have called our attention to many physiological details characterizing inversion. Hering for instance notes that changes of light and shade necessarily tend to produce such illusion, and he mentions the acceleration of changes by practice. Helmholtz notes the influence of passiveness of the eye in the production of inversion.

II. Two different tendencies are to be noticed in physiological theories : the first regards perspective figures as produced by changes of accommodation, the other regards them as produced strictly by movement of the eye. Necker ${ }^{1}$ after having denied that inversion could be an effect of will or accident selected accommodation as its principal cause.

By this he understands the relation existing between different points of the observed object and the central and peripheral parts of the retina. The part of the figure that is perceived with the fovea is estimated as nearer to the eye, the point observed by the peripheral parts of the retina as more remote.

Plausible as this theory appears, it does not take into account one of the principal phenomena of inversion, the absence of this illusion in certain cases of binocular and monocular vision. Secondly, Necker's theory is not to be considered as an explanation of the phenomena of accommodation, for we know that the same relations between the observed object and the special parts of the retina exist quite apart from accommodation.

Many years afterwards Loeb explained inversion by the same theory of accommodation, but he used it in its real significance, namely as innervation of the eye. His theory touches unquestionably one of the most important conditions of inversion, but it does not explain all the associated phenomena. For instance Loeb says that we obtain the impression of concavity in a perspective figure if the innervation removes the point of fixation, or that we have the impression of convexity by bringing the eye nearer to the figure. An easy experiment shows

1 Annal. der Physik u. Chemie.

2 Pfuegers Archiv, 1886, 411, 274. 
however that once we perceive a durable inversion we can change the accommodation successively and the image of inversion stands still. It is often necessary to open the second eye in order to escape the persistent illusion.

The most recent of physiological theories is that of W. Wundt recorded in his work on geometric-optical illusions. Inversion according to Wundt ${ }^{1}$ is caused by determined movement, consequently by change of points and lines of fixation. Wundt says : "The image of the retina ought to have a determined position if a perspective illusion is to appear; but the form of this illusion is entirely dependent on motion and direction of vision." Wundt indicates exactly what points and what lines ought to be fixed in order to obtain a concave or a convex form of inversion. Attentive observation proves, however, that if these movements can actually facilitate inversion they are not absolutely necessary to its appearance. It escapes the attention of Wundt that we can observe different forms of inversion as well if we fix only one and the same point or the same line of the figure.

We have yet to mention the experiments of Professor Mach, 2 of Vienna, which were applied chiefly to solid objects. They constitute the largest contribution yet made to our knowledge of the sum of the phenomena of inversion. Thus he observes the inversion produced by change of intensity in light and shade, or by different inclinations of the object, and he describes the phenomena of movement which accompany inversion. But Mach having described all the sedetails does not explain the cause producing them.

III. As for the special psycho-physical theories, which make use of the known facts in the functioning of the nervous system, they are mostly connected with more or less ingenious psychological hypotheses that have contributed little to the solution of the question. Such is for instance the theory of N. Lange, which explains inversion with the help of attention and of apperception. Lange ${ }^{3}$ has otherwise the merit of first trying to measure the rapidity with which the inversion occurs.

${ }^{1}$ Die geometrisch-optischen Täuschungen.

${ }^{2}$ Bcitrage zur Analy'se der Empfindungen, 86, and Wiener Sitzungsberichte Math.-natumiss. Classe, 54.

${ }^{3}$ Phil. Stud, Band IV., 405, I887. 
Because of the lack of a definite solution of the problem of inversion, a series of new experiments seemed necessary.

I. The first object of these experiments was to ascertain the degree of rapidity with which we perceive inversion in plane figures. Internal observation has already permitted us to suppose this rapidity greater than the rate of respiration, but slower than that of the pulse. Consequently the investigation was made in such a way as to observe the relation between the rapidity of inversion and the frequency of the pulse.

The result that was obtained by the graphic method, while the ex-

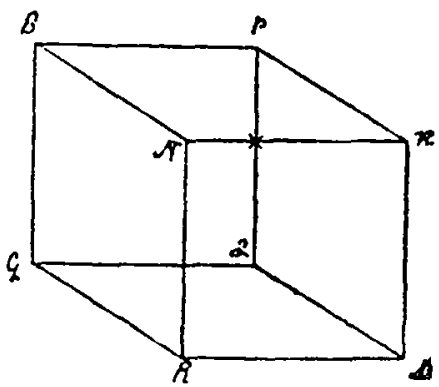

Fig.1. perimenter regarded intensely a plane figure (Fig. I made of copper wire), proved on the one hand that synchronism between

TABLE I. (Observer A).

\begin{tabular}{|c|c|c|c|c|c|c|c|c|c|}
\hline & Pulse & Inversion. & Pulse. & Inv & rsion & Pulse. & Inversion & Pulse & 'Inversion \\
\hline \multirow[t]{9}{*}{$30: \mathrm{X}$} & $2 I$ & 14 & 7 & 5 & $4 / X I$ & IO & 10 $12 / \mathrm{XI}$ & 16 & 20 \\
\hline & 39 & 27 & 37 & 23 & & $5 \mathrm{I}$ & 52 & 17 & 2 I \\
\hline & $\begin{array}{r}8 \\
17\end{array}$ & $\begin{array}{r}6 \\
12:\end{array}$ & $\begin{array}{l}17 \\
29\end{array}$ & $\begin{array}{l}13 \\
20\end{array}$ & & 28 & 27 & 21 & 26 \\
\hline & 9 & $84 / \mathrm{XI}$ & 47 & 46 & g/XI & $\begin{array}{r}51 \\
9\end{array}$ & $\begin{array}{l}30: \\
\text { I0: }\end{array}$ & & \\
\hline & 38 & 28 & 35 & $3 ;$ & & 38 & 39 & 1 & \\
\hline & 9 & 6 & $5^{8}$ & 65 & & 17 & IS & ? & \\
\hline & 7 & 5 & I5 & 16 & & 15 & 14 & & \\
\hline & 17 & 12 & 23 & $2 \mathrm{I}$ & & 56 & 60 & i & \\
\hline & 9 & 6 & 40 & 38 & & 42 & $47 !$ & ' & \\
\hline \multirow[t]{2}{*}{$2 / \mathrm{XI}$} & 7 & 5 & I2 & 12 & & I6 & IS & & \\
\hline & 37 & 23 & 20 & 29 & & 30 & 401 & 1 & \\
\hline
\end{tabular}

TABLE II. (Observer B).

\begin{tabular}{|c|c|c|c|c|c|c|c|c|}
\hline & Pulse. ' & Inversion. & Pulse & | Inversion & Pulse & Inversion & Pulse. & Iuversion. \\
\hline \multirow[t]{10}{*}{$30 / X$} & & 7 Io, XI & IO & Io & 8 & 8 & 5 & 5 \\
\hline & 4 & 4 & 10 & Io & 5 & 5 & 10 & IO \\
\hline & 5 & 5 & 6 & 6 & 17 & $17 !$ & I5 & I4 \\
\hline & 7 & 7 & 4 & 4 & 8 & 81 & 4 & 4 \\
\hline & $\delta$ & 8 & 17 & 8 & 5 & 5 & 3 & 3 \\
\hline & IO & Io & 5 & 3 & 8 & $8: 20 / \mathrm{XI}$ & 45 & 46 \\
\hline & 9 & 9 & IS & 10 & 4 & $4^{\prime}$ & 28 & 28 \\
\hline & 8 & 8 & 5 & 3 & 3 & 3 & I 7 & I3 \\
\hline & 9 & $9^{1}$ & IO & 7 & 5 & 5 & 7 & 7 \\
\hline & 7 & 7 & I 2 & I2 & 6 & $6 !$ & $3^{8}$ & 28 \\
\hline
\end{tabular}


the two does not exist, the rapidity of inversion being often inferior or superior to the rapidity of the pulse. It confirmed, on the other hand, what was already proved by internal observation, that these two rates of rapidity are very nearly the same,

In one person 124 inversions occurred in 174 pulsations; in another 260 inversions in 265 pulsations, and 313 inversions in 362 pulsations.

2. The fact that all persons engaged in these experiments declared positively that they had the feeling of an inward movement of the eye, even during an intense fixation of the indicated point of the figure, led to a question of a different kind, namely, what sort of movement it could be. With the help of an

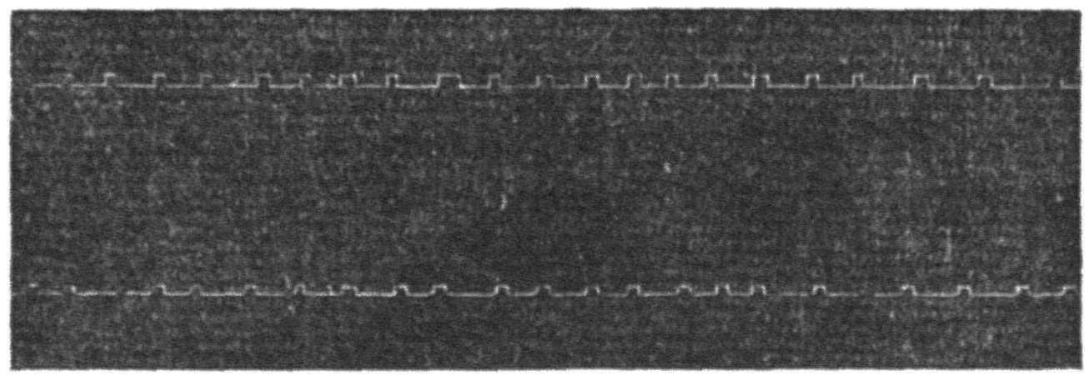

FIG. 2 .

ophthalmometer the eye of a myopic person ( 7 dyoptrics) was observed. The observations of light images of the external lens proved that every inversion produced in a plane figure is accompanied by a change of refraction. These changes are so distinct that it was not difficult at all to mark them graphically, when the observed person marked at the same time the changes of inversion, having fixed monocularly the hexahedron made of copper wire (Fig. 2). Of 543 double reactions obtained in this way, 343 were simultaneous notations of the changes of luminous images observed by the experimenter in the ophthalmometer and the notations of inversions by the observer of the hexahedron. In 132 cases this synchronism was dubious; in 68 the reaction failed completely, a fact which is explained by a decrease of attention on the part of the experimenter, or by a disturbing movement of the strongly lighted eye of the observed 
person. No series of these experiments lasted longer than a minute. These numbers proved distinctly the fact that the inversion of the plane figures can not be realized without a notable change of refraction.

3. But while the two preceding series of experiments were conducted on plane figures, it was necessary to complete them with experiments made on figures of three dimensions. To this end were prepared many figures of copper wire and many solid objects of small size.

But few trials were needed to show that the inversion appears as well in the case of solid objects as in that of plane figures. Almost any object can be used to illustrate the inversion ; for instance, a chair, a round or oval table, a lampshade, an umbrella - anything.

During these observations four main phenomena have been found to be characteristic of the inversions : $(a)$ changes in the intensity of light, in tone of color, and in the form of the observed object, $(b)$ the duration of the illusory image, $(c)$ the illusory movement, $(d)$ the inclination of the illusory image. But before we enter into the description of special phenomena of inversion a few words should be said concerning the difference of character between the inversion of plane figures and that of solid. Likewise something should be said about the difference in physiological conditions that go to produce inversions.

The difference between the plane and the solid figures consists in the fact that the first gives the sensation of two or more illusory forms, while the other presents but one. For instance : if we gaze intensely at the point of section of two lines of Necker's hexahedron, this plane figure appears immediately in three dimensions, one of its surfaces being inclined downward. This is the first illusory form. If we continue to fixate the same point of the figure, we perceive that this first illusory form has been transformed in such a way that we now see the same hexahedron lifted up. This is the second illusory form. Thus this inversion consists: (I) Of a passage of the normal sensation of a plane figure to the sensation of the first illusory form, (2) of the passage of the first to the second illusory form. But if instead of a perspective design we used a figure of three dimen- 
sions (of copper wire), then, having fixed the same point, we obtain only one form of illusion. Namely, if we observe an inclined surface of the figure we perceive the illusion as lifted up. If on the contrary we observe one that is elevated we have the illusion of an inclined figure. In both cases the illusion presents to the eye the passage of a real into an illusory sensation.

In the consideration of this difference of character in the two inversions we will distinguish, in what will follow, the inversion with one illusory form from the inversion with two such forms. The inversion which consists in the passage of a normal sensation to an illusory sensation will be called real-illusory inversion; the inversion which is the passage of one to the second illusory form will be called the pure-illusory inversion. This distinction is very important, because the physiological conditions necessary to produce both are not quite the same. To obtain a real-illusory form of inversion in any object or figure, cited above, it is sufficient to fix intensively and binocularly one point of the figure and presently to cover one eye with the hand in such a way that the eye remains in the greatest possible tranquillity. The inversion will not fail to appear in these conditions, and the degree of the passivity of the eye will decide the rapidity with which the inversion occurs. This effect can still further be facilitated by inhibition of all efforts to perceive the real dimensions of the object. The case is not the same in the inversion with double illusory form in plane figures. When the gaze is fixed in the same way they show the inversion marked by transition from a real to an illusory sensation, but they do not produce the passage of one to the other purely illusory inversion, unless there is either a movement of the ocular globe (Wundt's method) or a change of accommodation (Necker and Loeb) confirmed by the ophthalmometric observations of this essay. But by following either of these methods we can easily evoke both the forms of inversion above mentioned.

We now return to the description of the four kinds of phenomena, mentioned above, that characterize inversion.

(a) In the first place we have to do with the changes in the intensity of light, the shade of color, and the form of the observed object. All these details can easily be followed by using 
a visiting card in the same way as it was used by Mach. If this card, being first bent in the middle, is fixated monocularly at a point of its concave surface, we perceive it immediately as an illusory convex card. The illuminated part of the figure is now much more brilliant, the shadow deeper than it was in the moment preceding the inversion, or, strictly speaking, in the moment preceding the closing of the eye.

The shadowed part changes at the same time in color, passing through various tones, from gray or brown to yellowishgreen. This is especially noticeable if we change the position of the head without altering the point of fixation. Given the same conditions the form of the card is modified again.

(b) The real-illusory image acquires a certain duration, difficult to maintain in the plane figure, but very easy in the figure of three dimensions. So for instance the inversion of a concave cone, made of copper wire, does not disappear when observed monocularly, even or when the observer retires and re-approaches a distance of a few meters; or when the point of fixation is changed, or when the observer lays down or takes up his glasses, or even when the observer closes the eye for 2-5 seconds. As soon as the eye is opened the inversion reappears again.

(3) Illusory movement is the third characteristic phenomenon of inversion. It can be observed in both the plane and the solid figure. If an illusory image attains a certain duration in the consciousness, nothing is easier than to put it in an illusory motion. This result can be obtained by quietly changing the position of the head in any direction. The retinal image then changes exactly as it would change if the observed object were in movement. If we observe for instance the illusory image of a concave cone while the gaze is fixed on the central point of the figure, the illusory image will be like that in (a) Fig. 3. As soon however as the position of the head is directed to right or left, upward or downward, the image will appear in quite other perspective, such as $(b)$ and $(c)$.

Supposing now this movement is very slow and progressive. Then the sensation given by one point of view will join with that given by another point of view, etc.; we perceive the ob- 
ject in a synthesis of successive phases, consequently in what seems continuous morement. But if the illusion is to be perfect, it is necessary to change the angle of vision carefully and

b)

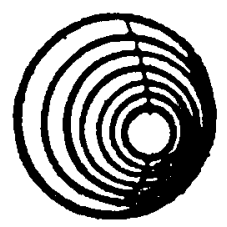

a)

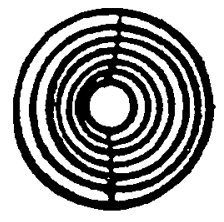

FIG. 3. c)

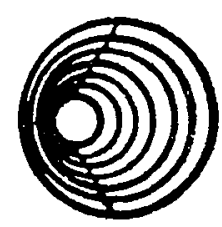

systematically, the gaze being strictly vis- $\mathfrak{a}-v$ is to the observed figure. That is to say, the line that joins the fovea with the fixed point of the object must be the radius of a circle in which the head moves. The ciliary muscles should be entirely passive. (In order to facilitate this circular movement, a circle was made on the floor with chalk around the table which sustained the figure.) Under these conditions all the perspective phases of the illusory image display themselves freely, from the first front view to the extreme right or left view, where the illusory image attains the maximum of abbreviation, before it disappears completely. This passage from the maximum abbreviation of the image to complete disappearance is very characteristic, because once coming to this maximal point our attention finds itself in a moment of doubt, whether the observed image is an illusion or a reality. But having advanced a little in the same circular direction we are reassured on this point. We then feel a kind of start in the eye produced by the disappearance of inversion and particularly of the illusory movement and the apparition of the real object remaining in the same place as before.

(c) The illusory movement in the plane figures (of copper wire) can easily be observed if one acquires a certain facility in preserving a durable inversion. So, for instance (Fig. 4), if the observer places himself at point (a) (nearly $60^{\circ}$ from the line $\left.b-b_{1}\right)$ of the circular line that surrounds the'Necker's plane figure of copper wire, he has to fix monocularly the point marked 
with a small cross and then to cover the second eye with the hand. He will obtain immediately the illusory image, namely, the illusion of a lifted hexahedron.

Having succeeded in making this illusion durable we have to follow very slowly and quietly the circular way from $a$ to $b_{1}$. The image of inversion passes during this time through a series of perspective phases, but, strange to say, these changes do not develop themselves in the same way throughout the arc.

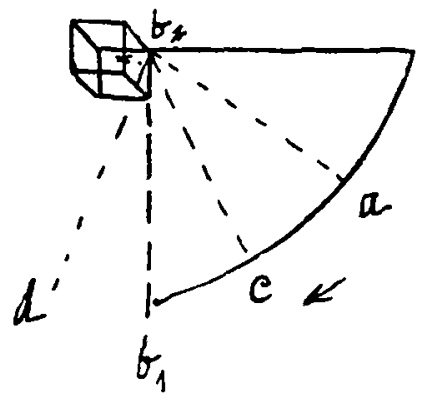

FTG. 4 .

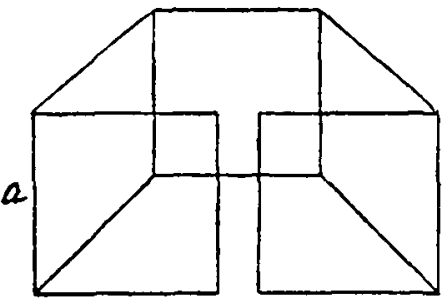

FIG. 5.

From $a$ to $c$ we observe the well-known changes of perspective phases, but from $c$ to $b_{1}$ the perspective phases disappear at once and we are startled with an image of the entire plane figure that displays a movement of rotation around its axis, which axis is formed by one of the lateral sides produced. Now the entire figure moves like a door on its hinge. This movement propagates itself without interruption if we continue the movement of the head forward and downward between $c$ and $b$. During these observations the figure appears continually as displaced, and this displacement is determined by the point held by the eye. If the eye is for instance in the point $c 30^{\circ}$ from the line $b-b_{1}$ the figure takes the direction of $d-b_{1}$ and so forth.

We can perceive the same illusion in many other figures, such as Fig. 5 (which must be fixated in the middle point of a lateral line, as $a$ ).

(d) There remains now the description of a most important phenomenon of inversion, namely, the inclination of the illusory image. This inclination is again in direct connection with 
the position of the eye in reference to the observed object. If one fixates binocularly and intensely a bent visiting card in its central point for instance, in such a way that the line joining the fixed point of the object with the fovea forms a horizontal radius of a circle, he obtains after having covered the second eye with the hand an illusory superficial image which is nearly plane and without any inclination. We mark this point of departure in the movement of the head by the letter $x$ (Fig. 6). Immediately

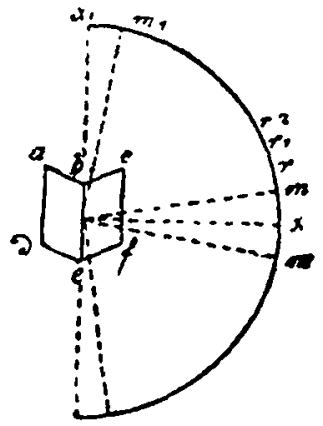

FIG. 6. if the eye is moved one degree up or down from the horizontal line, the same point being fixed, we obtain an illusory image of a slight inclination. These two points are marked on the figure with the letters $m-m$. If we continue now to move the head in the same circular direction from downward to upward, we come successively to the point $r, r_{1}, r_{2}$ of the circumference. At the same time the card changes its inclination in direct relation to the displacement of the eye. That means that to the movement of the head $a, a_{1}, a_{2}$, corresponds the inclination of the card in $r, r_{1}, r_{2}$. Once arrived to the point $x_{1}$ all illusion disappears. It attains its maximum at the point $m_{1}$. The eye traverses in this way $90^{\circ}$ from the outset of its displacement $(x)$ until it attains the point $x_{1}$; the card in the same time performs a movement of $180^{\circ}$ around its horizontal axis. Beginning from the point $m$ with a minimal inclination the card sinks more and more backwards, until all its points between $e$ and $b$ fall upon a horizontal line. The eye in this length of time traverses $45^{\circ}$, the card $90^{\circ}$. Then the card rises with the point $e$ until it assumes an upright position at $b$. Meanwhile the eye again traverses $45^{\circ}$, while the card has completed the $180^{\circ}$. But if before reaching the point $x_{1}$ we arrest the eye at the point of the maximum of inclination in the card $m_{1}$, we can now proceed in the opposite direction, and the illusory image will pass successively again through all the phases of inclination $r, r_{1}, r_{2}$, and come to the point $x$, at which the illusory image is nearly plane.

Similar changes of inclination can be made to appear by 
changing the point of vision in the right or the left direction. These phenomena may be corroborated by observing a concave card instead of a convex one. Here as in the preceding case are to be noticed: (I) The point at which the inversion is nearly plane and that at which it disappears completely $\left(x, x_{1}\right)$; The points of maximum and minimum inclination $r, r_{1}, r_{2}$.

These relations will be the same if instead of moving the head upon a circular line, we observe the object during its rotation around a horizontal axis. The direction however of the movement of the card will be changed: if we move it downward we see rotation in the opposite direction.

These experiments prove that the relation between the movement of the head and the inclinations of the illusory image is a fixed relation. The inclination of the illusory image is a function of the angle at which the eye observes the object.

Summarizing the results obtained from these experiments, we observe:

I. That the maximum of rapidity of inversion is nearly the same as the frequency of the pulse, though the relation is not functional.

2. Inversion in plane figures does not appear without a change of refraction.

3. We have to distinguish real-illusory inversion, and a pureillusory inversion which involves different physiological conditions.

4. Inversion appears in plane as well as in solid figures, and is accompanied by four distinct phenomena: (a) changes in the intensity of light, in the tone of color and in the form of the observed object; $(b)$ duration in the illusory image; $(c)$ movement of the illusory image; $(d)$ inclination, which is a function of the angle at which the eye observes the object. These observations as well as the corresponding experiments permit us to explain the phenomena of inversion in the following way.

It is certain that the cause of inversion consists in the relation between the observed object and the central and peripheral parts of the retina in which the image of the object produces itself. The points that fall upon the fovea are estimated more distinctly and consequently as nearer to the eye; those on the 
contrary that fall upon the peripheral parts, being less distinct, are considered as more remote.

These relations however are not sufficient to explain all the phenomena of inversion (its absence, for instance, in many cases of binocular or monocular vision) unless we take account of a certain irregularity of the vision when this illusion occurs.

The perception of an exterior object in its three dimensions can generally be the effect of binocular or monocular vision. In the binocular vision, as we know, the image of the retina, of the left eye for instance, is completed and controlled by the image of the right retina. Accordingly the coöperation of these two images, and at the same time the influence of accommodation and especially of convergence produce a normal representation of an object in space.

It is the same in monocular vision. We get no impression of the depth of an object with only one image of the retina, but only by two or more successive images of the same retina that complete and control each other. The representation of the object therefore is a result of two retinal images produced by the rays of light coming from two opposite directions.

It is quite different in the case of the illusion that we speak of. If we fix the object after closing one eye and by trying to immobilize it as much as possible, we suppress at the same time the control necessary to perceive the dimensions of the object. Closing one eye, we suppress the simultaneous image of the second retina; making the eye immobile during the monocular vision, we eliminate the successive image of the same retina. In this case therefore the part of the object that falls upon the central parts of the retina or upon its peripheral part are not completed and controlled by the simultaneous or successive images of it, the principal condition of a normal sensation. This stop of a regular progress of the visual function, suppressing the mutual compensation of images, is the principal cause of inversion.

It is clear now that a certain position of the eye is sufficient to produce one illusory form, the necessary correspondence between the parts of object and of the retina being given. But if we wish to pass from one to the other illusory form, it is 
necessary that the point of the object falling in the first case upon the central part of the retina should fall in the second upon the peripheral part and vice-versa. And this can not appear without the help of movement of the ocular globe or of changes of accommodation. For this reason it is sufficient to close the eye and to immobilize it to perceive the real-illusory inversion, but it is necessary to change the accommodation or the position of the eye if one attempts to pass from one to another illusory form. But it is absolutely indifferent to the eye what means are chosen for producing inversion of a double form. It may be a change of accommodation, such as Necker and Loeb employed, or such reflex changes as were the case in our ophthalmometrical experiments, or the movement of the ocular globe used by Wundt. This is of no importance: any proceeding is acceptable which facilitates the passage of the rays from an object once on the central part to another on the peripheral part of the retina. It is indifferent to the eye again that this change is produced in a passive way, changing the relation between the object and the eye, or in an active way, by placing the eye in a necessary condition.

Three capital facts make for this theory: (I) The inversion appears even when the ciliary muscle is immobilized with atropin (experiment made by Loeb). (2) The inversion can be summoned binocularly every time that the eyes are in the condition to observe simultaneously two images. (This experiment was made by observing a remote point of a small chair made of copper wire. Two chairs were seen together with an inverted image.) (3) The duration of the illusory image that persisted notwithstanding the changes of accommodation or movement of the ocular globe.

Finally, this theory explains all the phenomena characteristic of inversion. (a) The rapidity of inversion that was graphically measured was, as is now easily shown, a measure of the passage from one to the other illusory form. And because this passage does not appear except through a movement of the eye or of a change of accommodation this rapidity consequently is proportional to the rapidity of these movements or to this change of accommodation. In reference to inversion in figures of three 
dimensions the rapidity ceases to be characteristic, because the illusory image in this case can attain any duration.

(b) Again the duration of the image can be explained without difficulty. Because the inversion of an illusory form consists in a kind of stopping of the normal function of the eye, there is no reason why it should not persist as long as this irregularity lasts. In accordance with this, if one closes one eye to call up an inversion in a plane figure and endeavors to stop the movement of the eye necessary to evoke the passage of one to another illusory form, he obtains a durable inversion in the plane figure again.

(c) In reference to the typical movement and inclinations of the illusory image it is clear after what has been said, that successive positions of the eye, produced passively by the movement of the head without change of relation between a certain part of the object and the central and peripheral part of the retina, involve a continual change in the perspective of the retinal images. There is produced in the eye, under the influence of these successive displacements of the retinal image, a phenomenon nearly identical with what appears in such apparatus as the stroboscope or the cinematograph.

The only difference between these two phenomena consists in this: the stroboscope, being in possession of perspective images of an object in motion that were photographed from a central point but in different radia of the circle, supplies the means necessary to call into consciousness the synthesis of these images. But the images that are produced successively in the retina while the eye is displaced in different points of the periphery of the circle by the movement of the head, are, so to speak, successively photographed at different angles for the retina to produce a synthesis of an object in movement. Accordingly we can say the eye represents in this condition a living stroboscope."

'The MS. of this article was received November Io, I905. - ED. 